\title{
Utility of WhatsApp media to promote judicious use of antimicrobials in resource-limited settings
}

\author{
Dhanya Dharmapalan', Jeetendra Bhagwatrao Gavhane ${ }^{2}$ \\ From ${ }^{1}$ Consultant, Department of Pediatric Infectious Diseases, Apollo Hospitals, Navi Mumbai, Maharashtra, India, ${ }^{2}$ Professor, Department of \\ Pediatrics, Mahatma Gandhi Mission Medical College and Hospital, Navi Mumbai, Maharashtra, India
}

\begin{abstract}
Background: Promotion of rational antimicrobial use among health professionals is one of the cornerstones of a successful antimicrobial stewardship program. Various educational tools available do not provide a regular supply of updated information and can be expensive and inconvenient for the busy health-care professionals in resource-limited settings. Materials and Methods: An academic activity was conducted in the form of a daily WhatsApp tip on "rational antimicrobial use" over 2 months by a local branch of an academic body for pediatricians based in Navi Mumbai, India. Results: Evaluation of this tool through an online questionnaire resulted in 116 responses. The geographical locations of responders were widely distributed in India. The scientific content was rated as "excellent" by 78\% and practical utility as "extremely helpful" by $73 \%$. This tool was a preferred option as second-order (22\%) and third-order (25\%) of choices, among other educational tools such as textbooks, conferences, workshops, and training modules. Conclusion: Digital media in the form of WhatsApp can be an inexpensive tool for education for rapid mass coverage, provided that the scientific content is brief for daily assimilation and maintained at superior quality. It can be used for promoting evidence-based practices among health professionals, especially in resource-limited settings.
\end{abstract}

Key words: Antimicrobial stewardship, Children, Pediatrics, Survey, WhatsApp

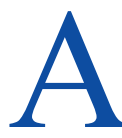
ntimicrobial stewardship program in resource-limited settings aims at building the best-practices capacity of health-care professionals regarding the rational use of antimicrobials [1]. Evidence-based practices help to reduce the irrational use of antimicrobials and improve the outcome of treatment among the patients. There are several educative tools by which the health-care professionals can stay updated with the latest recommendations. Textbooks, journals, workshops, conferences, training modules are some of these educative interventions which help to enrich the knowledge of the practicing medical professional. The World Health Organization has recently advocated the use of digital interventions based on mobile devices to address challenges of the health system [2]. These devices also have the potential to serve as a platform for an educational intervention for health-care professionals.

High rates of antimicrobial resistance reported in pediatric population in India highlighted the need to promote rational antimicrobial practices among health-care providers [3]. Therefore, there is an urgent need to develop an educational

\section{Access this article online}

Received - 03 August 2020

Initial Review - 29 August 2020

Accepted - 17 October 2020

DOI: $10.32677 / \mathrm{IJCH} .2020 . v 07.110 .004$ intervention in rational antibiotic use which is fast and qualitybased, can have large coverage, and cost-effective in resourcelimited settings. We evaluated the platform of a social media, WhatsApp on mobile devices for this purpose.

\section{METHODS}

In January 2020, a local branch of an academic body for pediatricians, based in Navi Mumbai and lead by the second author, conceived the idea of sending daily a tip on rational antimicrobial use to its members using WhatsApp. The first author, trained in pediatric infectious diseases, took on this responsibility to research current best practices and daily wrote a short tip (100150 words) on diagnosis and management of pediatric infectious diseases. The WhatsApp tips, 60 in total, were posted regularly from January 15, 2020, to March 20, 2020. Within a short period, these tips also got circulated in various WhatsApp groups and Email groups across the country. This practice was suspended in March when coronavirus disease-19 pandemic discussion took precedence on all WhatsApp groups.

Before stopping the activity, a link to a short online questionnaire, embedded in the tip, was sent. The questionnaire consisted of

Correspondence to: Dhanya Dharmapalan, 01, Satpura, Anushaktinagar, Mumbai - 400 094, Maharashtra, India. E-mail: drdhanyaroshan@gmail.com

(C) 2020 Creative Commons Attribution-NonCommercial 4.0 International License (CC BY-NC-ND 4.0). 
eight questions with multiple-choice answers: (1) Duration of professional experience; (2) area of specialty; (3) whether from urban or rural area; (4) the State of practice; (5) scoring of utility of the tool in practice; (6) rating of scientific content of the tool; (7) order of preferences of tools which keeps the user most updated on rational use of antimicrobials; and (8) suggestions of additional topics. We present our evaluation of this new tool of education, done electronically using the response to the feedback form.

\section{RESULTS}

The online survey rendered 116 responses. The majority had professional experience of $<10$ years $(34.5 \%)$, closely followed by between 10 and 20 years $(31.9 \%)$, greater than 30 years $(18.1 \%)$ and $20-30$ years $(15.5 \%)$. Most of the respondents were from the urban sector $(88 \%)$, while rest was from rural sector $(12 \%)$. The respondents belonged to various parts of the country, but majority were from the State of Maharashtra (56\%) followed by State of Gujarat (18\%). Three respondents belonged to Middle East countries: Dubai, Bahrain, and Kuwait. Of the respondents, $85.3 \%$ were general pediatricians, $5.2 \%$ pediatric intensivists, $3.4 \%$ belonged to other pediatric subspecialties, and $3.4 \%$ catered only to adults, while $2.6 \%$ to both adults and children.

The scientific content was rated as "excellent" by $78 \%$, "above average" by $19 \%$. The practical utility was recorded as "extremely helpful" by $73 \%$, "very helpful" by $24 \%$, and "somewhat helpful" by $2 \%$ of respondents. The final question was regarding the order of preference of five educative tools which help in practice to keep most updated on the rational use of antimicrobials. The results showed that the majority (34\%) preferred textbook as first-order preference (Fig. 1). The tool using WhatsApp featured prominently as second-order (22\%) and third-order (25\%) choice compared to other tools.

\section{DISCUSSION}

The results indicated that this tool appealed to all professional age groups, especially health-care providers who had recently started practice. The tool had reached practitioners across the country and also outside India indicating its wide geographical coverage.

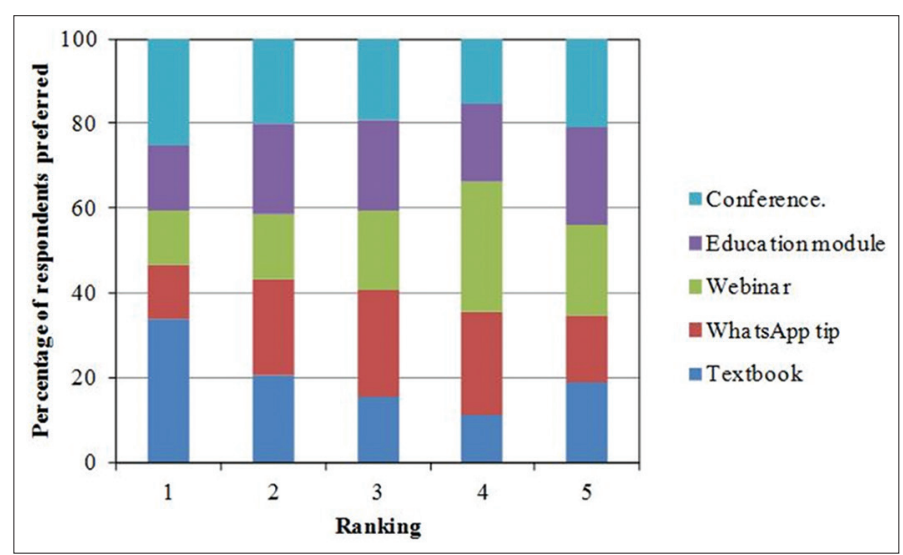

Figure 1: The order of preference for each education tool recorded by the survey responders (1: most preferred to 5: least preferred)
Although majority was from pediatric specialty, respondents included those catering to adults highlighting the scope of this tool in academic groups of other specialties. The high rating of scientific content and its practical utility, as indicated by the results were the reasons for the popularity of this tool.

WhatsApp has been evaluated as an effective learning tool among groups meant exclusively for education and mostly conducted by a moderator in a hospital/university setting [4]. To the best of our knowledge, this is a pioneering study evaluating its application for knowledge dissemination regarding antibiotic use among practicing pediatricians. At present, India is the biggest market for WhatsApp, with about 400 million monthly active users [5]. The success of this tool on WhatsApp was probably due to the high quality of short scientific content which could be assimilated within a short time compared to the time taken for using other educative tools and due to consistency. The choice of this digital tool in the second- and third-order of preference by maximum respondents indicates its growing importance as an additional source of easy and reliable information provided that the scientific content is maintained at a superior quality.

This digital tool provides time flexibility and convenience and is least expensive compared with other tools. The information sent through WhatsApp needs to be verified for accuracy as it can act as a double-edged sword if information circulated is incorrect. Furthermore, since there is no control of its reach, errors are non-retractable; therefore, caution has to be exercised before sending such messages. Due to the above reasons, only qualified and experienced authors should be encouraged to use this media for educative purpose. It will be advisable to peer review such messages well in advance by an expert team while adopting this digital tool for education.

This study has some limitations. Since this was a voluntary participation in an online survey, there could be a possible non-respondent bias which can affect the results of the study. Furthermore, being a research based on voluntary participation in a limited period, the sample size might not be a true representative of all the users of this tool.

\section{CONCLUSION}

There is a great need to reach to a wide population of health-care professionals in a cost-effective, the rapid manner for delivering good quality and updated information which can serve to use antibiotics rationally and improve patient management. WhatsApp tool can provide an easy and convenient educative tool for regular dissemination of brief, easy to assimilate scientific information by academic bodies to improve antimicrobial stewardship in resource-limited settings.

\section{ACKNOWLEDGMENT}

We acknowledge the support of Dr. Bakul Parekh, President, Indian Academy of Pediatrics and Dr. Vijay Yewale, Director, Dr. Yewale's Multispeciality hospital for Children, Navi Mumbai. 


\section{REFERENCES}

1. World Health Organization. Antimicrobial Stewardship Programmes in HealthCare Facilities in Low-and Middle-Income Countries. Geneva: World Health Organization; 2019. Available from: https://www.apps.who.int/iris/bitstream/han dle/10665/329404/9789241515481-eng.pdf. [Last accessed on 2020 Jun 30].

2. World Health Organization. WHO Guideline: Recommendations on Digital Interventions for Health Systems Strengthening. Geneva: World Health Organization; 2019.

3. Dharmapalan D, Shet A, Yewale V, Sharland M. High reported rates of antimicrobial resistance in Indian neonatal and pediatric blood stream infections. J Pediatric Infect Dis Soc 2017;6:e62-8.

4. Coleman E, O'Connor E. The role of WhatsApp ${ }^{\circledR}$ in medical education; a scoping review and instructional design model. BMC Med Educ 2019;19:279.

5. Singh M. WhatsApp Reaches 400 Million Users in India, Its Biggest Market; 2019. Available from: https://www.techcrunch.com/2019/07/26/whatsappindia-users-400-million. [Last accessed 2020 Jun 30].

Funding: None; Conflicts of Interest: None Stated.

How to cite this article: Dharmapalan D, Gavhane JB. Utility of WhatsApp media to promote judicious use of antimicrobials in resource-limited settings. Indian J Child Health. 2020; 7(10):412-414. 